\title{
Short communication: Sensory analysis of a kefir product designed for active cancer survivors
}

\author{
K. O’Brien, ${ }^{\star}$ C. Boeneke, ${ }^{*}$ W. Prinyawiwatkul, $\dagger$ J. Lisano, $\ddagger$ D. Shackelford, $\ddagger$ K. Reeves, $\ddagger$ M. Christensen, $\ddagger$ \\ R. Hayward, $\ddagger$ K. Carabante Ordonez,† and L. K. Stewart $\ddagger^{1}$ \\ *School of Animal Sciences, and \\ †School of Nutrition and Food Sciences, Louisiana State University Agricultural Center, Baton Rouge, LA 70802 \\ ¥School of Sport and Exercise Science and the University of Northern Colorado Cancer Rehabilitation Institute, Greeley, CO 80639
}

\section{ABSTRACT}

Kefir is a fermented milk product that is a good source of protein and health-promoting bacteria. It has the potential to improve recovery from exercise and the health and well-being of cancer survivors. The purpose of this study was to explore cancer survivor attitudes about and acceptance of a kefir recovery beverage made from cultured milk, whole fruit, natural sweeteners, and other natural ingredients. Kefir was made by inoculating and fermenting milk with kefir grains. The kefir was then mixed with a fruit base and given to cancer survivors $(\mathrm{n}=52)$ following a bout of exercise. Participants evaluated the acceptability of the beverage samples (overall appearance, aroma, taste, mouthfeel, and overall liking) using a 9-point hedonic scale, and they evaluated the smoothness using a 3-category just-about-right scale (not enough, just about right, and too much). They also expressed their physical and psychological feelings about the beverage using a 5 -point scale $(1=$ not at all to $5=$ extremely $)$ and indicated their purchase intent using a binomial (yes/ no) response. The health benefits of kefir were then explained, and participants sampled a second beverage (the same product), answering the same questions related to overall liking, feeling, and intent to purchase. We used a paired Student's t-test to compare beverage liking and emotion scores before and after participants learned about the health benefits of kefir. Data are presented as mean \pm standard deviations. The beverage scored significantly higher for overall liking after the health benefits were explained $(6.5 \pm 1.8$ and $7.0 \pm 1.7$ out of 9 before and after the explanation of health benefits, respectively). Participants showed a high intent to

Received November 16, 2016.

Accepted February 12, 2017.

${ }^{1}$ Corresponding author: Laura.Stewart@unco.edu purchase before they learned about the health benefits (75\% of participants indicated an intent to purchase, and $89 \%$ after they learned about the health benefits). The beverage received high scores overall and, except for an improvement in overall liking, we observed no significant differences in physical and psychological feelings before and after participants learned that it contained kefir and had potential health benefits. We found the beverage to be acceptable for consumption by cancer survivors, and the majority of participants showed an interest in purchasing for after exercise.

Key words: cancer survivor, consumer liking, emotion, exercise, kefir

\section{Short Communication}

Regular exercise plays an important role in improving cardiorespiratory fitness, muscular strength, and feelings of fatigue in cancer survivors during and after treatment (Repka et al., 2014; Repka and Hayward, 2016). Ingestion of enough fluids, protein, carbohydrates, and electrolytes after exercise is also necessary to ensure rapid recovery and repair of muscle tissue (American Dietetic Association et al., 2009). Milk has emerged as an ideal post-exercise beverage (Roy, 2008), and milk and dairy products have been linked to survival in people with cancer (Elwood et al., 2008). Unfortunately, many people with cancer experience digestive upset due to treatment (Omlin et al., 2013), and they may be wary of incorporating dairy products into their diet.

Kefir is a fermented milk product that is a source of protein, health-promoting bacteria, and carbohydrates after exercise. Documented health effects attributed to the consumption of kefir include improved lactose use, anticarcinogenic activity, control of intestinal infections, and improved milk flavor and nutritional quality (de Oliveira Leite et al., 2013). Studies evaluating the use of probiotics in physically active people have demonstrated maintenance of gastrointestinal function 
and health after exercise by reducing the frequency and severity of gastrointestinal issues, including cramps, nausea, bloating, and diarrhea (West et al., 2009). In fact, the combined effects of probiotics and exercise have demonstrated not only maintenance of gastrointestinal function and health, but also attenuation of immunosuppressive and cytotoxic effects, reduced susceptibility to illness, and enhanced resistance to upper respiratory tract infections (West et al., 2009; Ghoneum and Gimzewski, 2014). A recent study has suggested that the consumption of kefir may provide effects such as enhanced immune response and reduced inflammation in healthy athletes (O'Brien et al., 2015), but no studies have explored the potential of kefir for exercise recovery in active cancer survivors.

Regardless of the health-promoting benefits of a product, consumer taste preferences are most often the determining factor in accepting a new food as a regular part of the diet (Aggarwal et al., 2016). Fermented dairy foods typically score high in sensory tests, but most consumers prefer samples with the lowest acidity (Bayarri et al., 2011). Still, many enjoy the pronounced acidity and complex flavor profile associated with traditional kefir. The objective of this study was to explore cancer survivors' attitudes about and acceptance of a dairy beverage made from kefir, fruit, natural sweeteners, and other natural ingredients and flavorings. We hypothesized that cancer survivors would find the beverage more acceptable after they had been told about its health benefits.

A kefir beverage that met the American College of Sports Medicine guidelines for recommended nutrition after endurance and resistance exercise (American Dietetic Association et al., 2009) was developed and manufactured at the Louisiana State University Creamery. Pasteurized cow milk ( $4 \%$ fat) was fermented in 5 -gallon, lidded, stainless-steel milk pails by inoculation with approximately $30 \mathrm{~g}$ of kefir grains (Cultures for Health, Sioux Falls, SD) per 3.8 L of milk. The milk was allowed to ferment at $25^{\circ} \mathrm{C}$ for approximately $24 \mathrm{~h}$, or until a $\mathrm{pH}$ of 4.6 was reached. The kefir was then refrigerated until shipment. A fruit base - which included frozen strawberries, fresh bananas, honey, natural bourbon vanilla $1 \times$ concentrate, and stevia powder containing $95 \%$ total steviol glycosides - was processed separately and shipped on ice along with the kefir to the University of Northern Colorado Cancer Rehabilitation Institute (UNCCRI). After the kefir and fruit base were combined, 29.6-mL samples of the product were portioned into clear plastic containers with snapon lids and stored on ice for approximately $1 \mathrm{~h}$ before consumption. Nutritional analysis of the products and ingredients was performed using The Food Processor
Nutrition and Fitness Software (ESHA Research, Salem, OR). The composition of an 8-oz serving was 193 calories, $4.5 \mathrm{~g}$ of fat, $58 \mathrm{~g}$ of carbohydrate, $2.5 \mathrm{~g}$ of fiber, $27 \mathrm{~g}$ of sugar, $9 \mathrm{~g}$ of protein, and $68 \mathrm{mg}$ of sodium.

For the consumer test, all procedures were approved by the University of Northern Colorado Institutional Review Board. Subjects were male and female cancer survivors over 18 years of age who were currently undergoing or had completed surgical intervention, chemotherapy, radiation therapy, immunotherapy, hormonal therapy, stem cell, gene, or bone marrow transplantation, or other types of unconventional treatment, and were also current participants in the UNCCRI exercisebased cancer rehabilitation program. After participants reviewed the study procedures and provided informed consent, they completed a paper-and-pencil survey that included demographic questions (age, sex, and race), as well as questions related to their post-exercise recovery approach. The survey took no longer than $10 \mathrm{~min}$ in a quiet room at UNCCRI. Then, each participant completed a sensory evaluation that consisted of 2 samples (sample 1, sample 2) of the same beverage; the evaluation also lasted no longer than 10 min. During this phase, participants were seated in a large conferencestyle room at UNCCRI that was illuminated with natural fluorescent lights. Participants were briefed on the specific questions (with a focus on sensory attributes and their meanings) and on sample handling during the evaluation. Participants then consumed sample 1 and were asked to evaluate the acceptability of the beverage in the following order: overall appearance, overall aroma, overall taste, mouthfeel, and overall liking. Ratings were conducted using a 9-point hedonic scale $(1=$ dislike extremely, $5=$ neither dislike nor like, $9=$ like extremely; Peryam and Pilgrim, 1957). Participants were also asked to rate the intensity of mouthfeel and smoothness using the just-about-right (JAR) scale, which consisted of 3 categories $(1=$ not enough, $2=$ just about right, $3=$ too much). Then, they were asked to express their physical and psychological emotions/ feelings related to 13 terms, of which 12 were positive (accepted, active, calm, comforted, energetic, good, happy, healthy, refreshed, satisfied, steady, and social), and 1 was negative (disgusted), using a 5 -point scale (1 $=$ not at all, $2=$ slightly, $3=$ moderately, $4=$ very much and $5=$ extremely; King et al., 2010; King et al., 2015). The terms were preselected using an online survey that was delivered using Compusense 5, version 5.6 (Compusense Inc., Guelph, ON, Canada). Then, participants were asked to indicate their purchase intent using a binomial (yes/no) response (Sae-Eaw et al., 2007). After this phase, bottles of spring water and unsalted crackers were made available to allow participants to 
cleanse their palates. During this time, researchers also used the following phrases to inform participants that "the beverage contained kefir" and that "kefir improves the digestibility of milk and has been linked to improving intestinal flora and immune function." Participants then consumed sample 2 and were asked to answer the acceptability, physical and psychological emotions/feelings, and purchase intent questions again.

Group means and standard deviations were calculated for all liking and emotional responses. Overall liking and emotion scores were analyzed using a 2-related sample $t$-test to determine significant differences between responses before (sample 1) and after (sample 2) learning that the sample contained kefir and the associated health benefits of kefir. Correlations between overall liking and the hedonic values of appearance, aroma, taste, and mouthfeel were assessed via Pearson's r. A chi-squared test was used to compare purchase intent for sample 1 and sample 2. All data were analyzed using JMP (SAS Institute Inc., Cary, NC). Significance was generally set at $P<0.05$. However, to avoid inflation of the type I error rate, the significance criterion in the emotion terms was adjusted to 0.0038 ( $\alpha=0.05$ divided by 13 emotion terms).

Participants $(\mathrm{n}=52$; men $\mathrm{n}=22$, women $\mathrm{n}=30)$ averaged $60 \pm 29 \mathrm{yr}$ of age (range: 31 to $89 \mathrm{yr}$ ). Three participants were currently in treatment (radiation, chemotherapy, or both). Participants averaged $22.8 \pm$ 27.6 mo (range: 3 to $156 \mathrm{mo}$ ) from cancer diagnosis. All participants were in a structured exercise program at least twice per week. Overall liking of the beverage scored $6.5 \pm 1.8$ and $7.0 \pm 1.7$ out of 9 before and after the explanation of health benefits, respectively (Figure 1). Beverage attributes were measured after tasting sample 1, and average scores ranged from $6.0 \pm 2.0$ (aroma) to $7.1 \pm 1.6$ (mouthfeel). These scores were related to subjects "liking slightly to liking moderately." The average smoothness JAR score for this beverage was $2.0 \pm 0.2$, suggesting a "just about right" or close to ideal quality (Figure 2). The beverage received moderate to high scores for positive feelings (accepted, active, calm, steady, comforted, energetic, social, good, happy, healthy, refreshed, and satisfied), with average scores ranging from 2.8 (slightly) to 3.8 (moderately). Low scores for the negative feeling of disgusted (sample 1: $1.3 \pm 0.9$ and sample 2: $1.3 \pm 0.8$ ) indicated that on average participants were "not at all" disgusted by the beverage. We observed no significant differences in sensory liking and emotion/feeling scores between samples 1 and 2. We observed positive correlations between the overall liking and other sensory attribute liking scores for both samples 1 and 2, with the highest correlation values between overall liking and taste liking (Table 1). We also observed a high intent to purchase before par-

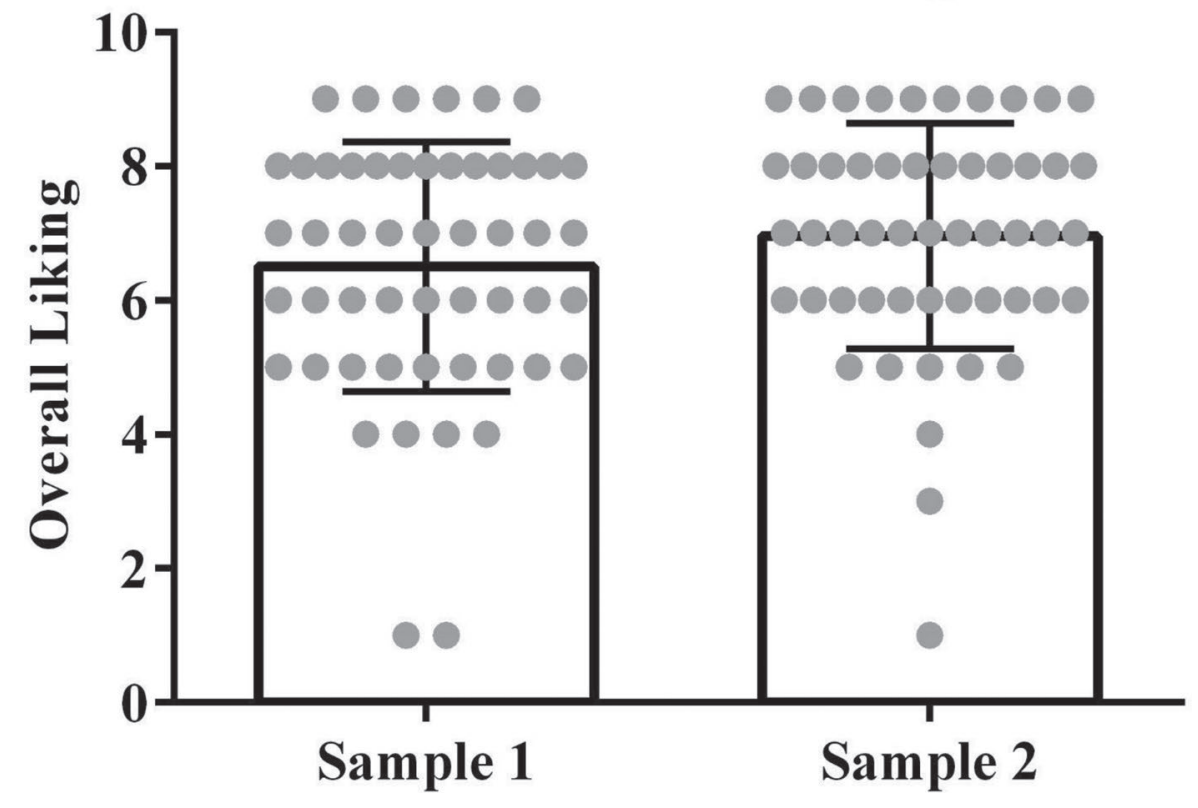

Figure 1. Overall liking of the kefir beverage before (sample 1) and after (sample 2) learning that it contained kefir and the health benefits associated with kefir. Data based on a 9-point hedonic scale are presented as individual data points (gray circles) and mean \pm SD. ${ }^{*}$ Significant difference $(P<0.05)$ between sample 1 and sample 2 


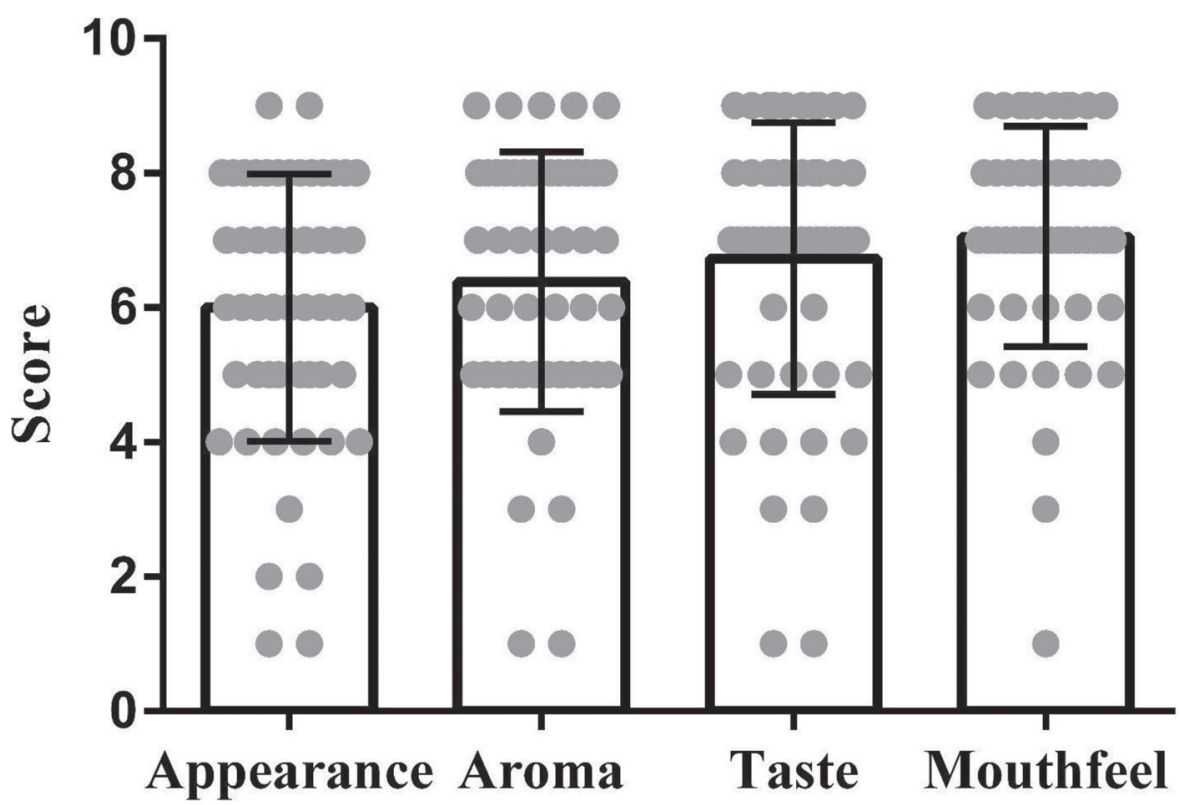

Figure 2. Sensory liking scores for appearance, overall aroma, overall taste, and mouthfeel of the kefir beverage based on a 9-point hedonic scale before the explanation (sample 1) of the health benefits of kefir. Data are presented as individual data points (gray circles) and mean \pm SD.

ticipants about the health benefits: 39 out of $52(75 \%)$ indicated a desire to purchase after sample 1, and this number increased significantly $(P=0.01)$ to 46 out of $52(88.5 \%)$ after sample 2 .

A possible health halo associated with smoothie beverages may have been associated with initial high overall scores. Although participants were not initially informed that they were drinking kefir, they knew that they were participating in a "smoothie" study.

The results of this study suggest that kefir may be acceptable to physically active cancer survivors after exercise. This is important information, because providing access to kefir may allow cancer survivors to enjoy the benefits of milk without stomach upset. This beverage also offers promise as a practical means of enhancing gut and immune function in this population.

Table 1. Correlations for overall liking scores of sample 1 and sample 2 with respect to the 9-point hedonic values for appearance, aroma, taste, and mouthfeel

\begin{tabular}{lcc}
\hline & \multicolumn{2}{c}{ Overall liking } \\
\cline { 2 - 3 } Sensory attribute & Sample 1 & Sample 2 \\
\hline Appearance & $0.682^{* * *}$ & $0.334^{*}$ \\
Aroma & $0.561^{* * *}$ & $0.403^{*}$ \\
Taste & $0.802^{* * *}$ & $0.551^{* * *}$ \\
Mouthfeel & $0.729^{* * *}$ & $0.45^{* * *}$ \\
\hline
\end{tabular}

, ${ }^{* * *}$ Correlation is significant at the ${ }^{*} 0.05$ or ${ }^{* * *} 0.001$ level ( 2 tailed).

\section{ACKNOWLEDGMENTS}

We thank the cancer survivors for their participation in this study. We also thank the staff at UNCCRI and the Louisiana State University Creamery for their support of this project.

\section{REFERENCES}

Aggarwal, A., C. D. Rehm, P. Monsivais, and A. Drewnowski. 2016. Importance of taste, nutrition, cost and convenience in relation to diet quality: Evidence of nutrition resilience among US adults using National Health and Nutrition Examination Survey (NHANES) 2007-2010. Prev. Med. 90:184-192.

American Dietetic Association, Dietitians of Canada, American College of Sports Medicine, N. R. Rodriguez, N. M. Di Marco, and S. Langley. 2009. American College of Sports Medicine position stand. Nutrition and athletic performance. Med. Sci. Sports Exerc. 41:709-731.

Bayarri, S., I. Carbonell, E. X. Barrios, and E. Costell. 2011. Impact of sensory differences on consumer acceptability of yoghurt and yoghurt-like products. Int. Dairy J. 21:111-118.

de Oliveira Leite, A. M., M. A. Miguel, R. S. Peixoto, A. S. Rosado, J T. Silva, and V. M. Paschoalin. 2013. Microbiological, technological and therapeutic properties of kefir: A natural probiotic beverage. Braz. J. Microbiol. 44:341-349.

Elwood, P. C., D. I. Givens, A. D. Beswick, A. M. Fehily, J. E. Pickering, and J. Gallacher. 2008. The survival advantage of milk and dairy consumption: An overview of evidence from cohort studies of vascular diseases, diabetes and cancer. J. Am. Coll. Nutr. 27:723S-734S.

Ghoneum, M., and J. Gimzewski. 2014. Apoptotic effect of a novel kefir product, PFT, on multidrug-resistant myeloid leukemia cells via a hole-piercing mechanism. Int. J. Oncol. 44:830-837.

King, S. C., H. L. Meiselman, and B. T. Carr. 2010. Measuring emotions associated with foods in consumer testing. Food Qual. Prefer. 21:1114-1116. 
King, S. C., J. Snow, H. L. Meiselman, J. Sainsbury, B. T. Carr, D. McCafferty, D. Serrano, M. Gillette, L. Millard, and Q. Li. 2015. Development of a questionnaire to measure consumer wellness associated with foods: The WellSense Profile (TM). Food Qual. Prefer. 39:82-94.

O'Brien, K. V., L. K. Stewart, L. A. Forney, K. J. Aryana, W. Prinyawiwatkul, and C. A. Boeneke. 2015. The effects of postexercise consumption of a kefir beverage on performance and recovery during intensive endurance training. J. Dairy Sci. 98:7446-7449.

Omlin, A., D. Blum, J. Wierecky, S. R. Haile, F. D. Ottery, and F. Strasser. 2013. Nutrition impact symptoms in advanced cancer patients: Frequency and specific interventions, a case-control study. J. Cachexia Sarcopenia Muscle 4:55-61.

Peryam, D. R., and F. J. Pilgrim. 1957. Hedonic scale method of measuring food preferences. Food Technol. 11(Suppl.):9-14.

Repka, C. P., and R. Hayward. 2016. Oxidative stress and fitness changes in cancer patients after exercise training. Med. Sci. Sports Exerc. 48:607-614.
Repka, C. P., B. M. Peterson, J. M. Brown, T. L. Lalonde, C. M. Schneider, and R. Hayward. 2014. Cancer type does not affect exercise-mediated improvements in cardiorespiratory function and fatigue. Integr. Cancer Ther. 13:473-481.

Roy, B. D. 2008. Milk: The new sports drink? A review. J. Int. Soc. Sports Nutr. 5:15.

Sae-Eaw, A., P. Chompreeda, W. Prinyawiwatkul, V. Haruthaithanasan, T. Swonsichon, J. E. Saidu, and Z. Xu. 2007. Acceptance and purchase intent of US consumers for nonwheat rice butter cakes. J. Food Sci. 72:S92-S97.

West, N. P., D. B. Pyne, J. M. Peake, and A. W. Cripps. 2009. Probiotics, immunity and exercise: A review. Exerc. Immunol. Rev. $15: 107-126$ 